Didáctica. Lengua y literatura

ISSN-e: 1988-2548

\title{
Principios didácticos para la enseñanza de vocabulario en español como lengua de aprendizaje del alumnado inmigrante
}

\author{
Marta Sanjuán Álvarez ${ }^{1}$; Cristina del Moral Barrigüete ${ }^{2}$
}

Recibido: 3 de junio de 2016 / Aceptado: 22 de enero de 2019

Resumen. La comprensión de la naturaleza de la competencia léxica debe servir de guía para enfocar su enseñanza de una manera más sistemática. En este artículo se establecen unos principios didácticos generales a partir de los diversos componentes que la integran, enfocados a una enseñanza más eficaz del léxico del español como segunda lengua del alumnado inmigrante en el contexto escolar, atendiendo a sus necesidades de aprendizaje. El objetivo es reforzar la adquisición del léxico con objetivos, contenidos y estrategias metodológicas claras que incluyan actividades sobre los procesos de comprensión, almacenamiento y recuperación para el uso progresivo del vocabulario académico en español. Para concluir este artículo, se ofrece una secuenciación didáctica de actividades para el desarrollo de la comprensión lectora y la competencia léxica, dirigida a alumnos inmigrantes, a partir de un texto escolar de Primaria.

Palabras clave: español como L2, alumnado inmigrante, enseñanza y aprendizaje del léxico, competencia léxica, procedimientos de enseñanza del léxico.

\section{[en] Educational principles for the teaching of vocabulary in Spanish as the language of instruction for immigrant students}

\footnotetext{
Abstract. Understanding the nature of lexical competence should serve as a guide to focus teaching in a more systematic way. In this paper, general didactic principles are established, based on the various components that involve this competence, focused on a more effective teaching of the vocabulary of Spanish as a second language for immigrant students in the school context, according to their learning needs. The objective is to reinforce their lexical acquisition with clear objectives, content and methodological strategies, that include activities on comprehension, storage and recovery processes for the progressive use of academic vocabulary in Spanish. To conclude this paper, a didactic sequence of activities for the development of reading comprehension and lexical competence, aimed to immigrant students, is offered, based on a text from primary school textbook.

Keywords: Spanish as L2, immigrant students, teaching and learning vocabulary, lexical competence, vocabulary teaching procedures.

1 Universidad de Zaragoza. Departamento de Didáctica de las Lenguas y de las Ciencias Humanas y Sociales. Grupo de investigación ECOLIJ.

E-mail: msanjalv@unizar.es

2 Universidad de Zaragoza. Departamento de Didáctica de las Lenguas, Facultad de Educación

E-mail: crismora@unizar.es
} 


\section{[fr] Principes didactiques pour l'enseignement du vocabulaire de l'espagnol en tant que langue d'apprentissage pour les étudiants immigrants}

Résumé. Comprendre la nature de la compétence lexicale devrait servir de guide pour aborder son
enseignement de manière plus systématique. Dans cet article, des principes didactiques généraux sont
établis à partir des différentes éléments qui le intégrent, dirigés vers un enseignement plus efficace du
lexique de l'espagnol en tant que seconde langue des élèves immigrants dans le contexte scolaire, et
répondant à leurs besoins d'apprentissage. L'objectif est de renforcer l'acquisition du lexique avec des
objectifs, contenus et stratégies méthodologiques clairs, comprenant des activités sur les processus de
compréhension, de stockage et de récupération pour l'utilisation progressive du vocabulaire académique
en espagnol. Dans la dernière partie, en guise de conclusion, une séquence didactique d'activités pour
le développement de la compréhension à la lecture et de la compétence lexicale, destiné aux élèves
immigrants, est proposé à partir d'un manuel d'école primaire. Mots-clés: l'espagnol comme langue seconde, élèves immigrants, enseignement et apprentissage du vocabulaire, compétence lexicale, procédés pour l'enseignement du vocabulaire

Sumario: 1. Introducción. 2. El currículo de español como L2 del alumnado inmigrante en el contexto escolar. La importancia del léxico. 3. Estrategias de enseñanza y aprendizaje del léxico en español como L2 vehicular. 3.1. Objetivos. 3.2. Contenidos. 3.2.1. Los procesos de comprensión, almacenamiento y recuperación para el uso. 3.3. Aspectos metodológicos. 4. A modo de ejemplo. 5. Conclusión. 6. Bibliografía.

Cómo citar: Sanjuán Álvarez, M.; del Moral Barrigüete, C. (2019). Principios didácticos para la enseñanza de vocabulario en español como lengua de aprendizaje del alumnado inmigrante, Didáctica. Lengua y literatura, 31, 99-116.

\section{Introducción}

Podemos considerar el proceso de aprendizaje del español por el alumnado inmigrante como un recorrido que va desde el desconocimiento total de la lengua, suponiendo que partimos de un nivel cero, hasta un aprendizaje del español equiparable al que hacen los alumnos españoles en su lengua materna. Como es evidente, la etapa inicial hasta la adquisición de unas competencias comunicativas básicas suele ser mucho más rápida en un contexto de inmersión social (hablamos entonces de L2) que cuando la lengua se aprende exclusivamente como asignatura de Lengua Extranjera (LE). El apoyo escolar que se da a esta etapa inicial del aprendizaje, con las aulas de español, protocolos de acogida, etc., contribuye, sin duda, al éxito en estas fases iniciales de la adquisición. Ahora bien, lo que está aún por resolver es cómo se realiza y facilita la transición entre las habilidades comunicativas básicas y las propias del dominio académico de la lengua.

El aprendizaje académico se apoya, esencialmente, en la capacidad de los alumnos para comprender y producir los textos orales y escritos propios de las diversas materias escolares: textos expositivos y argumentativos; textos literarios y textos relacionados con el aprendizaje literario; enunciados de problemas; textos que integran lenguajes verbales y no verbales, etc.

El objetivo fundamental del currículo de español como L2 en contexto escolar debería ser, por tanto, el de facilitar y acelerar aquellos aprendizajes que pudieran servir para acercar y equiparar, en la medida de lo posible, las competencias lingüísticas 
académicas de estos alumnos inmigrantes con las de sus compañeros españoles de su grupo de referencia, momento en el cual los aprendizajes lingüísticos pasarían a ser los propios del currículo de Lengua castellana para cada etapa educativa. El enfoque comunicativo de estos currículos de Lengua castellana como lengua materna abarca todos los aspectos que conducen a la competencia comunicativa plena de un hablante: intenciones comunicativas diversas, tipos de textos variados (incluyendo los académicos y literarios), procesos psicolingüísticos complejos de la comprensión y expresión oral y escrita, conocimiento y reflexión sobre la lengua en sus aspectos lingüísticos y comunicativos, contenidos socioculturales, sociolingüísticos, literarios...

Son numerosos los estudios teóricos y empíricos que intentan delimitar el concepto de competencia léxica en LE, aunque las definiciones manifiestan cierta confusión terminológica y ciertas discrepancias entre sí (Jiménez Catalán, 2002). El Consejo de Europa (2002, p. 108) define la competencia léxica como "el conocimiento del vocabulario de una lengua y la capacidad para utilizarlo".

La adquisición de la competencia léxica constituye un proceso complejo que incluye no solo la adquisición de unos contenidos léxicos, sino también unos procesos relacionados con la comprensión, la retención, el uso y la integración del vocabulario en redes asociativas de significados en el marco de esquemas de conocimiento culturales, de modo que aprender vocabulario de una lengua es inseparable de aprender la conceptualización del mundo que hace esa lengua.

\section{El currículo de español como $L 2$ del alumnado inmigrante en el contexto escolar. La importancia del léxico}

Los currículos de tipo funcional, que toman las funciones comunicativas como eje del aprendizaje del español como LE constituyen, sin duda, una referencia indispensable. Ahora bien, resuelven solo parcialmente los objetivos y contenidos necesarios para el aprendizaje del español como L2 del alumnado inmigrante en el contexto escolar, pues suelen quedarse en unos contenidos comunicativos sencillos, expresados con unos exponentes lingüísticos también sencillos, que vendrían a suponer, en definitiva, la adquisición de unas habilidades comunicativas básicas. La competencia comunicativa se concreta en la capacidad para comprender y producir discursos, por lo que los conceptos de texto y discurso son claves para el desarrollo del aprendizaje lingüístico. En el discurso confluyen todos aquellos factores significativos en un hecho comunicativo, los cuales "dejan su huella" en el texto emitido. Ello permite dar a las tareas de comprensión y producción de textos una dimensión pragmática y no solo lingüística, ligar las características lingüísticas de un texto a sus condiciones y contexto de producción, asociar de una manera significativa gramática y uso de la lengua; considerar, por tanto, la descripción gramatical como una herramienta para mejorar o facilitar el uso. Puesto que el trabajo con textos en el aula consiste en desarrollar habilidades de comprensión y producción de textos de muy diversas características, la progresiva complejidad de los textos utilizados supone, paralelamente, la progresiva complejidad de las habilidades psicolingüísticas, orales y escritas, implicadas en su comprensión y producción.

La incorporación de todos estos contenidos conduce a un planteamiento procesual o discursivo del currículo de español como L2. El objetivo final hacia el que debemos dirigir el aprendizaje lingüístico de este alumnado inmigrante es el de su 
plena incorporación a las tareas de aprendizaje del currículum escolar, para garantizar, en la medida de sus posibilidades, su éxito académico. Desde el punto de vista discursivo, los ejes principales para plantear un diseño curricular de español como L2 que facilite el periodo de transición entre los objetivos y contenidos comunicativos básicos y aquellos propios del español como lengua vehicular del aprendizaje en todas las materias del currículo, podrían ser los siguientes: 1) La comprensión, análisis (y posterior producción) de textos —orales y escritos - y estructuras textuales progresivamente más complejos, desde los propios de la comunicación interpersonal hasta los del ámbito académico, pasando por algunos de dificultad intermedia, como los de los medios de comunicación o determinados textos literarios. 2) El desarrollo de las destrezas de comprensión y expresión relacionadas con los textos académicos, con un énfasis especial en la comprensión del discurso oral del profesor, la comprensión lectora de textos expositivos y argumentativos, propios del aprendizaje escolar y la producción de los textos orales y escritos característicos del ámbito académico.

Pero un enfoque comunicativo, incluso de tipo discursivo, todavía resulta insuficiente para las necesidades comunicativas del alumnado inmigrante, que debe aprender el español como lengua vehicular. El aprendizaje de un vocabulario fundamental especifico de cada materia del currículo escolar (Ciencias Naturales, Geografía, Historia, Matemáticas, Literatura, Lengua, etc.) es un componente del currículo de español/L2 en el que habría que poner el énfasis desde el comienzo, ya que, sin duda, un desconocimiento casi absoluto del léxico de una materia convierte en muy remota la posibilidad de éxito académico en la misma. Para facilitar este aprendizaje se debe realizar un análisis de cuál es el vocabulario fundamental de cada área de conocimiento, así como una gradación de la dificultad en cuanto a su carácter básico o técnico.

En términos más generales, el léxico debe ser considerado como un eje principal en el aprendizaje del español como LE o L2. Por diversos motivos epistemológicos y didácticos, la enseñanza de este componente de la lengua se ha visto relegada a un estatus secundario, en favor de la gramática y, en general, se ha realizado de manera intuitiva, poco sistemática, sin unos materiales o un currículo suficientemente respaldados por la investigación acerca de cómo se configura la competencia léxica y qué medios didácticos parecen los más efectivos para que el alumno la adquiera. Es evidente que su didáctica entraña unas dificultades epistemológicas y metodológicas especiales: frente a la fonología o la sintaxis, el léxico no se deja fácilmente analizar y estructurar de manera lógica. Sin embargo, tanto los alumnos como los profesores y los investigadores reconocen la importancia central del vocabulario en el conjunto de las capacidades que se engloban en el saber comunicar en una lengua:

It is the experience of most language teachers that the single, biggest component of any language course is vocabulary. No matter how well the student learns grammar, no matter how successfully the sounds of L2 are mastered, without words to express a wide range of meanings, communication in an L2 just cannot happen in any meaningful way. And yet vocabulary often seems to be the least systematized and the least well catered for all the aspects of learning a foreign language (McCarthy, 1990, p. viii).

En el siguiente epígrafe se revisan algunas cuestiones relevantes acerca de la competencia léxica en ELE. Los diversos aspectos que se van considerando irán 
dando lugar a una serie de reflexiones que buscan su aplicabilidad a la enseñanza del español para alumnado inmigrante en el contexto escolar.

\section{Estrategias de enseñanza y aprendizaje del léxico en español como L2 vehicular}

Las principales decisiones que tiene que tomar el profesor que quiera favorecer el desarrollo de la competencia léxica del alumnado inmigrante en el contexto escolar tienen que ver con qué objetivos, qué contenidos, qué procedimientos metodológicos pueden ser más adecuados, atendiendo a sus necesidades de aprendizaje.

\subsection{Objetivos}

Dos objetivos son fundamentales para el aprendizaje léxico del EL2 del alumnado inmigrante (Sanjuán, 2008, p. 174):

1. Lograr una progresiva acomodación sociocognitiva desde el conocimiento del mundo inicial del alumno en su propia lengua hacia unos repertorios léxicoculturales característicos del español como lengua de aprendizaje. Ello supone la ampliación de dominios temáticos, comenzando con el universo de la vida cotidiana y progresando hacia otros dominios temáticos más especializados. Se trata de que el alumno avance desde los marcos generales del conocimiento hacia la adquisición y memorización de repertorios léxico-culturales, organizados por temas representativos del universo cultural del español y de los ámbitos temáticos escolares. Posteriormente, se profundizará en esos temas mediante un análisis cada vez más detallado de las distinciones léxicas en cada dominio considerado y mediante el estudio de micro-sistemas asociados a textos y contextos de uso del ámbito académico.

2. A la vez, habrá que trabajar para un enriquecimiento léxico que consista no solo en un progreso cuantitativo sino también cualitativo, lo que incluye dos procesos: un análisis cada vez más profundo de las distinciones léxicas en cada dominio considerado; pasar de los niveles concretos a la conceptualización abstracta.

Pero además, la idea de la adquisición del vocabulario como un proceso "desordenado", poco sujeto al control del profesor, implica la necesidad de proporcionar a los alumnos herramientas que les ayuden a organizar ese aprendizaje desordenado, a construir agrupamientos coherentes y significativos entre las palabras, a desarrollar los procesos de comprensión, retención y uso mediante estrategias metalingüísticas, metacognitivas, técnicas de autoaprendizaje, etc. Se trata, básicamente, de llevarlos a analizar las relaciones intralingüísticas y de orientarlos, en general, hacia un autoaprendizaje reflexivo (Sautermeister, 1989).

Esta visión del aprendizaje del léxico como un aprendizaje necesariamente autónomo, dado que el profesor podrá dedicar atención específica a un número muy reducido de unidades léxicas, se ha afianzado con el desarrollo del enfoque AICLE (Aprendizaje Integrado de Contenidos y Lenguas Extranjeras), que pone el énfasis en que los alumnos aprendan a aprender vocabulario. Los objetivos de enseñanza 
del léxico incluirían, por tanto, no solo la adquisición de unos determinados contenidos léxicos, más o menos prefijados, sino también el desarrollo de estrategias de autoaprendizaje. El profesor puede presentar y explicar vocabulario, pero en último término son los alumnos los que aprenden y encuentran la manera de expandir y organizar sus almacenes léxicos. Son ellos los que necesitan aprender a pensar, a razonar, a tomar decisiones meditadas, a responder en forma creativa y a construir una estructura a través de la cual interpretar el significado y la comprensión (Coyle, Hood y Marsh, 2010).

\subsection{Contenidos}

¿Cuántas palabras podemos enseñar en un curso? ¿Qué palabras de la lengua serán más útiles para los alumnos? ¿Qué palabras habría que seleccionar para cada etapa educativa? ¿Puede haber algún tipo de secuenciación que facilite el aprendizaje? ¿Se debe distinguir entre vocabulario receptivo y vocabulario productivo? ¿Debemos enseñar solo palabras o hay otro tipo de unidades léxicas en la lengua? Todos estos interrogantes abordan, en definitiva, la cuestión central de "qué vocabulario enseñar".

La lingüística nos proporciona datos acerca de la frecuencia de las palabras, su dispersión (variedad de diferentes textos o campos temáticos en los que aparecen) y disponibilidad (palabras que vienen antes a la mente asociadas a un tema), su carácter general o específico, así como del tipo de redes asociativas en el sistema y en el discurso (relaciones sintagmáticas, paradigmáticas, intratextuales). El Diccionario combinatorio del español contemporáneo (Bosque, 2005) es, en este sentido, una herramienta didáctica indispensable.

Además, son decisivos los factores motivacionales, puesto que el aprendizaje léxico es en gran medida un aprendizaje autónomo e implica una disposición del alumno a procesar en profundidad las palabras (Laufer y Hulstijn, 2001). Una selección interesante de temas, textos y técnicas que favorezcan el aprendizaje puede contribuir a crear esa motivación. Por tanto, los criterios lingüísticos deben matizarse con otros criterios psicolingüísticos: necesidades e intereses de los alumnos, cuántas palabras pueden asimilar y retener, qué vocabulario será previsiblemente receptivo y cuál puede convertirse en productivo (Palapanidi, 2013; San Mateo, 2013), qué factores influyen en la facilidad o dificultad de su aprendizaje.

Deberá tenerse en cuenta también que el aprendizaje del vocabulario es inseparable de los procesos de comprensión, retención y uso, que dan lugar a otro tipo de contenidos: estrategias metalingüisticas, metacognitivas, técnicas de autoaprendizaje, etc.

\subsubsection{Los procesos de comprensión, almacenamiento y recuperación para el uso}

La lingüística moderna nos ha facilitado la comprensión de algunos principios que regulan el comportamiento de las palabras en el sistema y en el discurso. Otros factores, de naturaleza psicológica, intervienen en cómo los hablantes, nativos o extranjeros, se "apoderan" de una parte más o menos extensa de ese vasto almacén.

Los procedimientos de un almacenamiento eficaz, indica Aitchison (1987), no tienen por qué coincidir con los de una activación eficaz. Es cierto que, para la didáctica del léxico, son fundamentales los tres estadios de procesamiento de las palabras: la comprensión, el almacenamiento y la recuperación (Martín, 1999; Baralo, 2001). 
Estos procesos están relacionados, pero no son idénticos. Puede ser, por ejemplo, que "sepamos" una palabra (la tengamos almacenada) pero no sepamos "recuperarla" (evocarla para el uso).

\section{La comprensión y el reconocimiento de las palabras}

Las muestras de lengua que se ofrecen a los alumnos (input) en el proceso de adquisición del léxico de una LE son, en un principio, básicamente orales. Después, el input escrito puede ser incluso más importante. Hay, por tanto, dos vías diferentes de percepción de las palabras: la forma fonológica y la ortográfica. Ellis (1995, p. 12) considera que el lexicón está formado por múltiples componentes e incluye la posibilidad de varios lexicones especializados (fonológicos, ortográficos, motóricos o de pronunciación, sintácticos, semánticos) para diferentes canales de la recepción y la producción.

Los procesos relativos a la comprensión y reconocimiento de las palabras nos conducen a dos cuestiones didácticas centrales: ¿qué tipo de input es mejor?, ¿cuánto input nuevo puede procesar la mente?

Aun valorando la importancia de la vía fonológica como mecanismo de adquisición del léxico, es indiscutible que la lectura constituye una de las principales fuentes de enriquecimiento léxico. Además, en las personas alfabetizadas la forma ortográfica de las palabras es, sin lugar a dudas, un componente de las entradas del lexicón mental. La presentación contextualizada parece imprescindible para un aprendizaje verdadero de las palabras, que incluye saber usarlas. La presentación aislada, asociada a una definición o explicación, puede facilitar la comprensión, pero no favorece su evocación en el uso. Posiblemente habría que combinar, por tanto, los beneficios que se obtienen de distintos tipos de input (oral y escrito; palabras aisladas y en contextos de uso). Por otra parte, no deberíamos desdeñar la importancia de muestras de lengua "informales" o input incidental (canciones, películas, televisión, etc.), en los que el vocabulario se adquiere de forma poco consciente (McCarthy 1990; Laufer, 2004; Agustín Llach, 2013).

Un aspecto importante de la comprensión y adquisición de vocabulario a través del contexto es ayudar a los alumnos a comprender el significado de las palabras desconocidas. Para ello, señala Nattinger (1988) que es imprescindible desarraigar en los alumnos estrategias de aprendizaje equivocadas como intentar entender todas las palabras del texto; tampoco hay que entender exactamente el significado de una palabra desconocida, puede ser suficiente con atribuirle un sentido general. Solo después de encontrar una palabra en múltiples contextos es cuando se accede a una comprensión más o menos completa de su significado. El diccionario es una herramienta necesaria para el alumno, pero el profesor puede ayudar a desarrollar otras técnicas para adivinar el significado y utilizar el diccionario como último recurso. Nattinger propone, por ejemplo, la enseñanza de estrategias metalingüísticas de inferencia a partir de las pistas contextuales, tales como el tema general; otras palabras del discurso (redundancias, anáforas, paralelismos); la estructura gramatical; la morfología (análisis de los prefijos, sufijos, composición, etc.); la entonación (habla) y la puntuación (escritura).

Otro aspecto que hay que tener en consideración para favorecer los procesos de comprensión es el de cuántas palabras nuevas puede procesar la mente. De una manera general, McCarthy (1990) opina que la proporción de palabras desconocidas/ 
conocidas no debe exceder de 1 cada 15 palabras en un texto de 750 palabras, aunque sobre este aspecto del input no hay una respuesta definitiva, ya que en la capacidad de asimilación influyen factores como el tipo de texto, longitud, propósito comunicativo, motivación, etc.

\section{El almacenamiento y la retención}

Aunque a veces nos referimos al conocimiento léxico como "diccionario mental", realmente el almacén mental de palabras es mucho más rico que un diccionario. Higueras (2000, p. 16) define este lexicón mental como un "almacén" inteligente en el que los elementos léxicos se encuentran interrelacionados de diferentes maneras. Frente a las limitaciones del diccionario, tanto Higueras como Lahuerta y Pujol (1996) utilizan la metáfora del “ordenador" — que permite búsquedas rápidas, múltiples referencias cruzadas entre palabras, posibilidades casi sin límites de almacenar gran cantidad de información-.

Para explicar cómo se relacionan las palabras en la mente se ha elaborado un modelo de "redes mentales", lo que significa un "sistema interconectado" (Aitchison, 1987, p. 72) en el que se entrecruzan diferentes tipos de asociaciones: fonológicas, ortográficas, semánticas, enciclopédicas, morfosintácticas, afectivas. Las redes conceptuales de los hablantes competentes van mucho más allá de la información semántica de las palabras y abarcan un conocimiento enciclopédico que las une al conocimiento del mundo. En síntesis: las palabras se almacenan y recuerdan en redes asociativas de diversos tipos (semánticas, formales, fonológicas, visuales) y se asocian también a los contextos en que se aprendieron. Además, la actividad mental ejerce una gran influencia en la memoria y hay relación directa entre "esfuerzo cognitivo" (cognitive depth de Craik y Lockhart, 1972. Cit. por Nattinger, 1988) y retención: cuanta más atención dedicamos al aprendizaje de una información, más retenemos. Otro aspecto de interés es que la memoria trabaja mejor con "segmentos de información" que con unidades aisladas; aunque esos segmentos sean más largos y contengan más información se retienen más fielmente que si se presentan simplemente en una lista.

Ellis (1995) sugiere que la retención del significado se acentúa mediante actividades de procesamiento en profundidad, mientras que los rasgos formales se adquieren, sobre todo, mediante la práctica. Cuanto más se trabaje con una palabra y más atención consigamos que preste cada alumno a la entonación, ortografía, categoría gramatical, pronunciación, etc., más probabilidad tendrá esa palabra de entrar al lexicón y formar parte de él.

Se han descrito numerosas técnicas para favorecer la retención a largo plazo en el aprendizaje holístico de una lengua:

- Aprendizaje memorístico por repetición, aunque esta técnica es solo válida en un estadio inicial de aprendizaje (Gairns y Redman, 1986).

- Técnicas mnemotécnicas (Gairns y Redman, 1986; Pressley, Levin y McDaniel, 1987; Nattinger, 1988) como mapas cognitivos, imágenes visuales, diagramas, fichas de vocabulario o escenarios mentales, que facilitan la memorización y evocación de conjuntos de palabras.

- Agrupamientos de palabras formales (por ejemplo, palabras con los mismos prefijos griegos o latinos, etc.); de familias léxicas (estimulan las asociacio- 
nes formales y semánticas); de combinaciones sintagmáticas (las palabras que aparecen en el entorno de una palabra ayudan a delimitar su significado, además de favorecer su retención); agrupamientos semánticos (palabras agrupadas en guiones de conocimiento que representan esquemas culturales de la lengua y se pueden combinar con representaciones visuales).

- Tareas significativas que incluyan algún tipo de procesamiento por campos semánticos o guiones de conocimiento y proporcionen un tema unificador que facilite la organización en la memoria; este tipo de tareas ayuda al alumno al análisis profundo y provoca un recuerdo más elaborado, intenso y duradero que la repetición oral, por ejemplo. Beck, McKeown y Omanson (1987) abogan por que los alumnos se centren en el significado de la palabra nueva e intenten integrar ese significado en las redes semánticas que ya poseen.

- Sistemas escritos de almacenamiento léxico como diagramas, ideogramas, árboles semánticos, diccionarios personales, etc. (Gairns y Redman, 1986; Grymonprez, 2000; entre otros).

Además de la mayor o menor profundidad del procesamiento cognitivo, la retención del vocabulario nuevo puede estar relacionada con factores afectivos del aprendizaje. La presentación del nuevo vocabulario en contextos significativos - con implicación en tareas que requieran una participación activa (tareas de interacción, de descubrimiento dirigido, de respuesta con una actividad, etc.) — favorecerá su adquisición.

\section{La recuperación para el uso}

La habilidad de recuperar las palabras para el uso es esencial. Sin embargo, podemos tener almacenadas muchas palabras que no usamos en nuestra expresión, pero sí reconocemos y comprendemos cuando las oímos o leemos. Hay que distinguir, pues, entre acceso receptivo al lexicón (decodificación) y acceso productivo (codificación).

$\mathrm{El}$ acceso receptivo supone unir los enunciados orales o escritos con las estructuras fonológicas y ortográficas almacenadas y sus correspondientes significados. Algunos alumnos pueden presentar dificultades en la discriminación de las palabras si desarrollan el hábito de una decodificación demasiado analítica de los elementos auditivos o visuales, en lugar de atender a la forma general. La decodificación eficaz se apoya decisivamente en el contexto, que delimita los caminos de activación semántica, es decir, qué acepción es la adecuada para una determinada forma léxica.

Las frases hechas y lexías complejas tienen una entrada léxica propia, puesto que se almacenan sin descomponer. Igualmente, las palabras compuestas y derivadas se almacenan independientemente de las palabras raíces, aunque los prefijos y sufijos podrían tener sus propias entradas, ya que parecen estar disponibles en los usos creativos del lenguaje (Aitchison, 1987); además, este tipo de palabras ayudará a desarrollar una mayor fluidez expresiva y receptiva (Lewis, 1993, 1997, 2000). Para Higueras (1997, pp. 46-48) enseñar combinaciones léxicas presenta múltiples ventajas: evita errores léxicos, ayuda a almacenar juntas en el lexicón palabras que se emplean como un bloque, se profundiza en el conocimiento de palabras ya presentadas y se mejoran notablemente las destrezas comunicativas.

El acceso productivo sigue el itinerario inverso: del significado a la forma, ya sea esta una palabra simple, compuesta, derivada, una combinación prefijada, una frase hecha, etc. 
La capacidad para recuperar las palabras está en estrecha relación con los sistemas empleados para su almacenamiento en el lexicón. Cuanto mejor estén organizadas las palabras en la mente, más fácil será su evocación. También son importantes las técnicas para ayudar a recuperar el vocabulario almacenado en el lexicón y estimular el uso de ese conocimiento almacenado animando a los alumnos a usar el vocabulario recientemente adquirido, aunque ello pueda llevarles a un cierto "riesgo expresivo" o a hacer uso de su competencia estratégica (Carter y McCarthy, 1988; Nattinger, 1988). Ahora bien, Liceras \& Carter (2009, p. 25) han comprobado que "la profundidad de conocimiento de las palabras es un factor fundamental para determinar el tipo de conexión mental que se va a producir. No hay correlación con las palabras poco conocidas pero sí con las bien conocidas."

San Mateo (2013) considera conveniente valorar el grado de dominio que precise el estudiante, pues en algunos casos será suficiente con realizar un ejercicio de reconocimiento para poder adquirir ciertas unidades léxicas, mientras que en otros habrá que practicar su uso productivo a través de actividades en las que se crea un contexto apropiado.

En cuanto a la cantidad de vocabulario que se puede enseñar (y que los alumnos pueden aprender), Lewis (1993, pp. 122-123) manifiesta que una metodología centrada en el profesor - con unas secuencias de enseñanza en las que el profesor controla de manera explícita todos los contenidos léxicos que van a ser objeto de aprendizaje - dificulta más que favorece ese proceso. Esta dificultad conduce a un planteamiento metodológico que combine los procedimientos directos con los indirectos, como se expone a continuación.

\subsection{Aspectos metodológicos}

El aprendizaje del léxico integra una gran diversidad de componentes complejos. Gairns y Redman (1986) conceden gran importancia a las estrategias dirigidas a la implicación de los alumnos en su propio aprendizaje para lograr mayor auto-motivación y hacerlos más responsables, al tiempo que permiten al profesor una mayor atención a las necesidades individuales. Se mencionan, por ejemplo, las actividades de discusión o resolución de tareas en pequeños grupos; enseñar el manejo del diccionario; enseñar a deducir el significado de las palabras a través del contexto; actividades de descubrimiento como encontrar algún tipo de categorización en una lista de palabras, etc.

Si aceptamos que la dicotomía gramática/vocabulario no es válida, como apuntaba Lewis (1993, p. 90), que el agrupamiento de palabras en secuencias léxicas (pedagogical chunking) ha de ser una actividad frecuente en clase, pues la combinación de segmentos ayuda a lograr fluidez verbal, o que el léxico, la morfología y la sintaxis son un continuum de aspectos interrelacionados, según uno de los postulados básicos de la lingüística cognitiva (Cuenca y Hilferty, 2000, p. 19), estaremos de acuerdo con que el tratamiento del léxico en el aula de español/L2 ha de integrar actividades relacionadas con las diferentes acepciones de las unidades léxicas (semántica), su estructura (lexicogénesis), las relaciones paradigmáticas (hiperonimia, sinonimia, antonimia, polisemia), las relaciones sintagmáticas (combinaciones, colocaciones, expresiones fijas) y el valor discursivo que implica el contenido que se comunica (Gómez Molina, 2003, p. 4). En función de este conocimiento complejo, Gómez Molina cree necesario el uso de una metodología que combine la presentación signi- 
ficativa del vocabulario en situaciones y contextos de uso con el desarrollo de estrategias de aprendizaje que permitan atribuir un sentido a las palabras y a las posibles asociaciones entre palabras.

El profesor debe, o al menos procurará, presentar todo el contenido léxico objeto de estudio en contextos identificables para que el significado de la expresión completa sea claro en relación con el discurso en el que ocurre (contexto y cotexto). Entre los factores que contribuyen al dominio de todas estas unidades léxicas están la selección de textos auténticos apropiados, así como el diseño de diversas y variadas actividades relacionadas con técnicas y estrategias que potencien la observación, categorización, uso, retención y fijación de dichas unidades en la comunicación diaria y profesional (Gómez Molina, 2003, p. 101).

Por otra parte, Coady (1997, pp. 275-281) ofrece una descripción detallada de los principales enfoques metodológicos de enseñanza del léxico, desde los más estrictamente indirectos (adquisición solo a partir de contextos significativos comprensibles) hasta los basados casi exclusivamente en la instrucción explícita o directos.

Los procedimientos indirectos (lectura, conversación, películas, etc.) son indispensables para aumentar cuantitativamente el conocimiento del léxico (sobre todo del receptivo) - ya que con las actividades de aprendizaje explícito solo se puede abarcar un número reducido de ítems léxicos-, pero también para adquirir los aspectos borrosos del significado de las palabras (connotaciones culturales, sociales, conocimiento enciclopédico), así como los aspectos relacionados con su comportamiento discursivo en los diferentes tipos de textos, tanto orales como escritos.

En una actividad de lectura, según Laufer \& Hulstijn (2001) se recordarán con mayor precisión: las palabras cuyo significado fue inferido correctamente antes que las que se explicaron con un sinónimo; las palabras que se buscaron en el diccionario antes que las que estaban explicadas al margen del texto; las palabras negociadas durante la actividad comunicativa antes que las no negociadas; las palabras que se utilizaron en tareas productivas antes que las que se usaron en tareas receptivas.

En el caso del alumnado inmigrante, se trataría, como ya se ha señalado, de establecer una secuencia de textos académicos graduados, en los que el léxico específico de cada materia fuera apareciendo también de manera graduada, con unos glosarios para facilitar su comprensión. Entre los múltiples beneficios de trabajar con textos auténticos, Swaffer (1985) y Swain (1995) mencionan: una mayor motivación para los aprendices; la posibilidad de que los textos completen los esquemas de conocimiento que puedan tener sobre un tema en particular o sus esquemas culturales; $y$, de manera específica, una mejora en el vocabulario, la gramática y las estructuras retóricas, que se adquieren en contextos significativos. Para Coyle $(1999,2005)$ en la metodología AICLE confluyen cuatro componentes que representan el modelo de las "cuatro ces": la cultura (culture) será la que vertebre la negociación de los contenidos (content), a través de la lengua meta (communication) y el desarrollo de habilidades concretas (cognition) que permitan una mejor comprensión del conocimiento del mundo.

La presentación indirecta de nuevas palabras a través de contextos de uso, única manera de abarcar un corpus amplio, se debe completar con otros procedimientos directos o de enseñanza explícita, que contribuyen a desarrollar una conciencia metalingüística sobre determinados aspectos del léxico. Algunas técnicas de instrucción directa ayudan al alumno, por ejemplo, a desarrollar estrategias de comprensión de las palabras desconocidas a través de las pistas contextuales, morfológicas, sintácticas, etc. 
Los procedimientos directos pueden contribuir, además, a que el alumno adquiera los aspectos estables del significado de las palabras, así como una comprensión de las relaciones léxicas sistematizables y de los principios que regulan el comportamiento y la formación de palabras, es decir, aquellos aspectos del léxico sancionados por la tradición cultural y normativa, presentes en diccionarios, gramáticas, etc.

Las actividades o ejercicios de enseñanza directa tratan de presentar las palabras y demás unidades léxicas agrupadas en torno a temas (para favorecer su integración en esquemas de conocimiento), asociaciones semánticas (campos semánticos, antonimia, sinonimia, hiponimia, metaforización, acepciones básicas y específicas, etc.), asociaciones formales (familias léxicas, prefijos, sufijos, procedimientos de composición), combinaciones típicas o frases hechas, etc.

Higueras (2007, pp. 41-42) valora los mapas semánticos como un recurso especialmente interesante, que pueden utilizar tanto el profesor como el alumno. Un buen mapa semántico tiene que contener todo tipo de unidades léxicas: palabras y unidades léxicas pluriverbales; sobre todo, no debe convertirse en una colección de sustantivos, sino que tienen que estar representadas todas las categorías gramaticales. Además, debe incluir elementos gráficos que favorezcan la retención y permitir la inclusión de nuevas palabras en cada categoría, así como la creación de nuevas ramas. Al prevalecer un criterio temático es más fácil que se utilicen esos recursos léxicos en actividades comunicativas que, a su vez, proporcionarán aprendizaje incidental de nuevas palabras o consolidación y ampliación de redes entre palabras conocidas.

Sanjuán (2008, p. 180) describe una serie de estrategias utilizadas por una docente de EL2 para reforzar explícitamente la comprensión implícita del vocabulario incluido en una tarea comunicativa que simula una situación auténtica y que incluye lectura, comprensión oral y expresión oral: 1) crear un contexto imaginario pero cercano a los alumnos para situar y ejemplificar el tema; 2) expandir el contenido léxico de una palabra o unidad léxica, bien mediante expansiones descriptivas a base de hipónimos, sinónimos, paráfrasis o circunloquios, bien introduciendo campos semánticos asociados y agrupando el vocabulario en un marco de conocimiento cultural que incluye comportamientos, acciones o lugares; 3) enfatizar con la entonación, o escribir las palabras clave en la pizarra; 4) dar una definición, ejemplificar los conceptos difíciles o explicar la etimología.

\section{A modo de ejemplo}

A continuación, se ofrece una secuenciación didáctica de actividades para el desarrollo de la comprensión lectora y la competencia léxica dirigida a alumnos inmigrantes en contexto escolar, cuyo nivel de español deseable al finalizar la etapa primaria ha de ser de B1. El texto ha sido extraído de un manual de Ciencias Sociales para 4. ${ }^{\circ}$ Primaria.

1. Objetivo: activar esquemas de conocimiento del mundo y esquemas de vocabulario relacionados con el texto, antes de su lectura.

Conversación dirigida por el docente:

a) ¿Sabéis qué es una inundación? ¿Qué clases de inundación puede haber? ¿Qué puede provocar una inundación? ¿Qué daños puede ocasionar (a las personas, a las casas, a la agricultura, etc.)? ¿Habéis visto alguna vez una 


\section{Lo posible: saber qué hacer en una inundación}

Los daños producidos por el desbordamiento del río Azul y la rambla Nabla se van solucionando: la carretera N-430 volverá a abrirse al tráfico después de reparar el asfalto. El parque de la ribera del río Azul sigue inundado. Sin embargo, ya han finalizado las obras de recuperación del patio del colegio. Sus alumnos volverán el lunes a las aulas.

Las noticias para los vecinos de las viviendas construidas en el cauce de la rambla no son positivas. Tras llevar seco varias décadas, su caudal subió espectacularmente y la violencia de sus aguas arrasó las casas.

Para prevenir estas situaciones, el ayuntamiento ha difundido a los ciudadanos estas recomendaciones.

Antes de la inundación: Durante la inundación:

- Preparar un kit de supervi- - Si estás en casa: apagar la vencia con radio, linterna, alimentos enlatados, mantas, botiquín...

- Tener a mano los teléfonos de emergencia, el seguro del hogar y establecer un punto de reunión familiar.

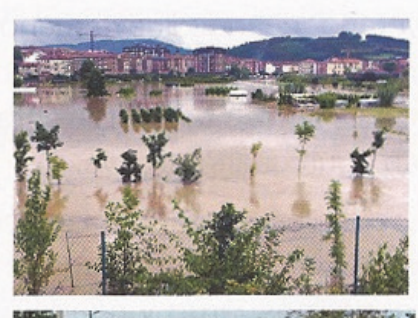
luz, el gas y el agua. Subir al lugar más alto y escuchar por la radio los consejos de las autoridades.

- Si estás en un vehículo: salir de él, evitar andar por las aguas en movimiento .

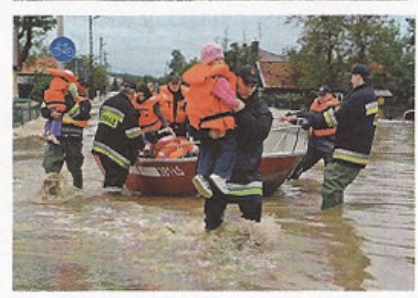

Fuente: Alfonso Talavera, E., Bellón Mena, A., Moral Santa-Olalla, A., Tapiador Pérez, C. (2015). Ciencias Sociales. 4. ${ }^{\circ}$ Primaria. Proyecto "Saber hacer". Sevilla: Grazalema/Santillana.

inundación? ¿Qué pueden hacer las personas cuando saben que va a ocurrir una inundación en su pueblo o ciudad?

2. Objetivo: hacer una lectura rápida del texto para fijarse en ciertos aspectos (títulos destacados tipográficamente, ilustraciones) y generar hipótesis sobre el contenido y la intención general del texto.

El profesor entrega el texto y deja que los alumnos le echen un vistazo en un tiempo breve. A continuación dirige el análisis de las ilustraciones y los títulos:

a) ¿Qué os ha llamado más la atención de este texto?

b) ¿Qué se ve en las ilustraciones? (El profesor anota en la pizarra el vocabulario que mencionen).

c) ¿Sabríais qué hacer en una inundación?

d) ¿Dónde puede haber aparecido este texto? ¿Cuál puede ser la intención de quien lo ha escrito? ¿Quién puede dar consejos e informaciones para saber qué hacer en una inundación?

e) Relaciona el título del texto (Lo posible: saber qué hacer en una inundación) con los subtítulos Antes de la inundación y Durante la inundación y anticipa el contenido de esa parte del texto que aún no se ha leído.

3. Objetivo: tras una lectura completa del texto, verificar o no las hipótesis previas y comprender las ideas principales. 
a) ¿Qué ha ocurrido? ¿Cuál es la noticia? ¿Qué es lo que ha producido la inundación?

b) ¿Qué daños ha provocado...? Si no lo recordáis, buscadlo en el texto.

- En el parque

- En la carretera

- En el patio del colegio

- En las casas

c) ¿Qué consejos da el Ayuntamiento a la población?

4. Objetivo: inferir por el contexto o por la estructura morfológica de las palabras el significado de las palabras desconocidas.

a) ¿Qué pueden significar estas palabras o expresiones tal como aparecen usadas en el texto? Intentad adivinar el sentido y buscad sinónimos cuando sea posible (en parejas). También os podéis fijar en si se parecen a otras que ya conocéis (por ejemplo, venir/prevenir).

\begin{tabular}{|c|c|}
\hline $\begin{array}{l}\text { - Rambla } \\
\text { - Desbordamiento } \\
\text { - Ribera } \\
\text { - Cauce } \\
\text { - Caudal } \\
\text { - Arrasó } \\
\text { - Prevenir }\end{array}$ & $\begin{array}{l}\text { - Kit } \\
\text { - Emergencia } \\
\text { - Seguro del hogar } \\
\text { - Punto de reunión } \\
\text { - Tener a mano } \\
\text { - Evitar andar } \\
\text { - Tras llevar seco } \\
\quad \text { varias décadas }\end{array}$ \\
\hline
\end{tabular}

5. Objetivo: recuperar para el uso las palabras nuevas adquiridas y expandir el contenido léxico en esquemas de conocimiento cultural.

a) Explicad para qué puede servir cada consejo que da el ayuntamiento sobre qué hacer antes y después de la inundación, y para qué puede servir cada objeto que se incluye en el kit de supervivencia.

b) Completad ese kit de supervivencia con otras cosas que creáis que podrían ser útiles.

c) Reconstruye el orden cronológico de los hechos que aparecen en esta noticia. ¿Qué ha pasado?

1. La carretera está cortada. No hay tráfico. No pueden pasar coches porque hay mucha agua.

2. Las obras del patio del colegio están terminadas.

3. Los alumnos podrán volver a clase el lunes.

4. Se ha desbordado un río y una rambla. El agua del río ha llegado a la ciudad.

d) ¿Y después? Investiga utilizando los recursos en internet (vídeos, noticias, etc.) qué pasa después de una inundación. Escribe un breve texto con la información que hayas leído.

6. Objetivo: ampliar el conocimiento del léxico mediante asociaciones semánticas, relaciones de hiponimia o esquemas de conocimiento propios del ámbito académico. 
a) Verbos del campo semántico de "reparar". Poned ejemplos de cosas o situaciones que podemos reparar, solucionar, coser, zurcir, pegar, restaurar, recuperar (por ejemplo, reparar el calzado).

b) Clases de ríos o maneras en que el agua puede ir canalizada. Unid las palabras de la izquierda con su definición:

\begin{tabular}{|c|c|}
\hline $\begin{array}{l}\text { 1. Arroyo } \\
\text { 2. Afluente } \\
\text { 3. Rambla } \\
\text { 4. Río } \\
\text { 5. Riachuelo } \\
\text { 6. Canal } \\
\text { 7. Acequia }\end{array}$ & $\begin{array}{l}\text { A. Río de poca agua, casi continuo. } \\
\text { B. Río secundario que desemboca o desagua en otro principal. } \\
\text { C. Suelo por donde corren las aguas de lluvia cuando llueve } \\
\text { mucho. } \\
\text { D. Río pequeño y de poco caudal. } \\
\text { E. Cauce artificial por donde se conduce el agua. } \\
\text { F. Zanja o canal por donde se conducen las aguas para regar. } \\
\text { G. Corriente de agua continua y más o menos caudalosa que va } \\
\text { a desembocar en otra, en un lago o en el mar. }\end{array}$ \\
\hline
\end{tabular}

c) Partes de un río. Buscad en el diccionario las palabras que aparecen en este dibujo sobre las partes de un río y poned donde corresponda las de la derecha:

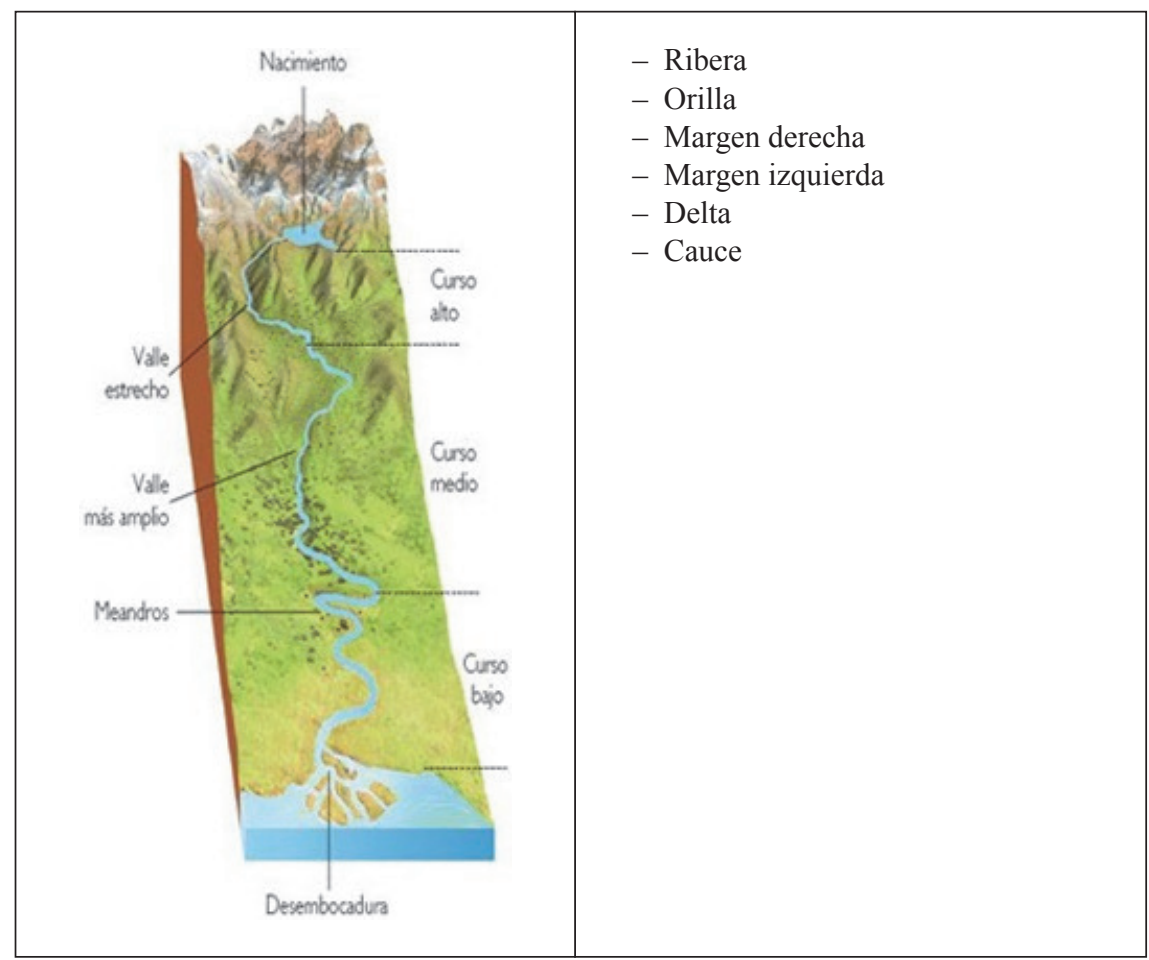

Fuente: CEIP José Saramago. Recuperado de http://elquintVopino5.blogspot. com/2018/10/partes-de-un-rio.html 


\section{Conclusión}

A lo largo de este trabajo se han presentado unos principios didácticos que pueden contribuir a una enseñanza más eficaz del léxico del español como segunda lengua del alumnado inmigrante. El léxico es un componente fundamental en el aprendizaje de una lengua, ya que sin palabras es casi imposible la comunicación, pero esa relevancia se acentúa aún más en el contexto del español como lengua de aprendizaje del alumnado inmigrante, ya que no está reforzada suficientemente la transición del uso comunicativo básico a unos usos académicos que requieren el dominio de un léxico complejo, poco habitual fuera del contexto escolar.

Con carácter general, hemos considerado la necesidad de abordar un enfoque metodológico discursivo para el aprendizaje del EL2 en el contexto escolar, que aborde el desarrollo de habilidades de comprensión y producción de textos cada vez más complejos y de características muy diversas dependiendo de las materias del currículo, lo que conlleva una progresión de las habilidades psicolingüísticas de comprensión y producción de textos orales y escritos. Además, resulta imprescindible la adopción de estrategias docentes específicas para facilitar el aprendizaje del vocabulario asociado a cada materia escolar y para acelerar la adquisición de las habilidades comunicativas propias del dominio académico de la lengua.

Todo ello se ha plasmado en una secuenciación de actividades para la explotación didáctica de un texto escolar del área de Ciencias Sociales, como forma de integrar el desarrollo de la competencia léxica del alumnado inmigrante en un enfoque discursivo más general característico del modelo AICLE.

En definitiva, sería bueno que los profesores de EL2 orientáramos la enseñanza del léxico hacia actividades didácticas que favorecieran la implicación cognitiva del alumno a la vez que su competencia léxica: tareas que estimularan la reelaboración de esquemas de conocimiento, el pensamiento y la organización mental, en lugar de la reproducción, la repetición o la simple traducción.

\section{Bibliografía}

Aitchison, Jean (1987): Words in the mind: An introduction to the mental lexicon, Oxford, John Wiley \& Sons.

Agustín Llach, María Pilar (2013): "Dinámica del aprendizaje incidental de léxico en lengua extranjera", en Revista Nebrija de Lingüistica Aplicada, 14 [en línea]: http://www.nebrija.com/ revista-linguistica/files/articulosPDF/articulo_526a6afcdce77.pdf [consulta: 28 mayo 2016]

Baralo Ottonello, Marta (2001): "El lexicón no nativo y las reglas de la gramática", en ELUA. Estudios de Lingüistica, Anexo 1, 23-38. [en línea]: http://dx.doi.org/10.14198/ ELUA2001.Anexo1.02 [consulta: 28 mayo 2018]

Beck, Isabel L., Margaret G. McKeown y Richard C. Omanson, (1987): "The effects and uses of diverse vocabulary instructional techniques", en The nature of vocabulary acquisition, Margaret G. McKeown y Mary E. Curtis, (Eds.). Hillsdale, Lawrence Erlbaum Associates, 147-163.

Bosque Muñoz, Ignacio (2005): Diccionario Redes: Diccionario combinatorio del español contemporáneo, Madrid, SM.

Carter, Ronald y Michael McCarthy (1988): Vocabulary and Language Teaching, Harlow, Longman. 
Coady, James (1997): "L2 vocabulary acquisition: A synthesis of the research", en Second Language Vocabulary Acquisition, Coady, James y Thomas Huckin (eds.), Cambridge, Cambridge University Press, 273-290.

Consejo de Europa (2002): Marco común europeo de referencia para las lenguas, Estrasburgo, Consejo de Europa/MECD/Instituto Cervantes.

Coyle, Do (1999): "Theory and planning for effective classrooms: supporting students in content and language integrated learning contexts", en Learning through a Foreign Language, Masih, John (ed.), London, CILT.

Coyle, Do (2005): CLIL: Planning tools for teachers. Nottingham, University of Nottingham.

Coyle, Do, Philip Hood y David Marsh (2010): CLIL. Content and language integrated learning, Cambridge, Cambridge University Press.

Cuenca, María Josep y Joseph Hilferty (2000): Introducción a la lingüística cognitiva, Barcelona, Ariel.

Ellis, Nick (1995): "Vocabulary Acquisition. Psychological Perspectives", en The Language Teacher, 19 (2), 12-16.

Gairns, Ruth y Stuart Redman (1986): Working with words: A guide to teaching and learning vocabulary. Cambridge, Cambridge University Press.

Gómez Molina, José Ramón (2003): “La competencia léxica en el currículo de español para fines específicos", en Actas del II Congreso Internacional de Español para Fines Específicos (CIEFE), 82-104. [en línea]: http://cvc.cervantes.es/Ensenanza/biblioteca_ele/ciefe/ pdf/02/cvc_ciefe_02_0009.pdf [consultado: 2 mayo 2018]

Grymonprez, $\bar{P}$ ol (2000): “El enfoque léxico y la enseñanza del español para fines específicos», en Mosaico. Revista para la promoción y apoyo a la enseñanza del español, (5), $16-22$.

Higueras García, Marta (1997): "Las unidades léxicas y la enseñanza del léxico a extranjeros", en REALE, 8, 35-49.

Higueras García, Marta (2000): "Favorecer el aprendizaje del léxico", en Boletín de ASELE, $23,13-18$.

Higueras García, Marta (2007): “Técnicas para la enseñanza del léxico” en Mosaico. Revista para la promoción y apoyo a la enseñanza del español, 20, 37-42.

Jiménez Catalán, Rosa María (2002): “El concepto de competencia léxica en los estudios de aprendizaje y enseñanza de segundas lenguas", en Atlantis, 24 (2), 149-162.

Lahuerta Galán, Javier y Mercé Pujol Vila (1996): “El lexicón mental y la enseñanza del vocabulario", en Segoviano, Carlos (coord.). La enseñanza del léxico como lengua extranjera: homenaje a Anton e Inge Bemmerlein. Iberoamericana, Vervuert Verlagsgesellschaft, 117-129.

Laufer, Batia y Jan Hulstijn (2001): "Incidental vocabulary acquisition in a second language. The construct of task-induced involvement", en Applied linguistics, 22 (1), 1-26.

Laufer, Batia (2004):'Focus on Form in Second Language Vocabulary Learning" en Conferencia plenaria en el 14 Congreso Internacional EUROSLA, San Sebastián, 8-11 Septiembre.

Lewis, Michael (1993): The Lexical Approach, Hove, Language Teaching Publications.

Lewis, Michael (1997): Implementing the Lexical Approach, Hove, Language Teaching Publications.

Lewis, Michael (2000): Teaching collocations. Further developments in the Lexical Approach, Hove, Language Teaching Publications.

Liceras, Juana y Diana Carter (2009): "La adquisición del léxico” en Manual de Lexicología, Madrid, Ariel. 
Martín Martín, Soledad (1999): "La revisión del concepto de vocabulario en la gramática de E/LE", en Didáctica del español como lengua extranjera 4, Lourdes Miquel y Neus Sans (coords.), Madrid, Fundación Actilibre/Colección Expolingua, 157-163.

McCarthy, Michael (1990): Vocabulary, Oxford, Oxford University Press.

Nattinger, James (1988): "Some current trends in vocabulary teaching", en Vocabulary and Language Teaching, Carter, Ruth y Michael McCarthy, Harlow, Longman, 62-82.

Palapanidi, Kiriakí (2013): "Conocimiento receptivo y productivo del vocabulario de LE", en Revista Nebrija de Lingüística Aplicada a la Enseñanza de las Lenguas, 7 (6). [en línea]: http://www.nebrija.com/revista-linguistica/files/articulosPDF/articulo_526a6b6c2ca3e.pdf [consultado: 22 mayo 2018]

Pressley, Michael, Levin, Joel R., McDaniel, Mark A. (1987): "Remembering versus inferring what a word means: Mnemonic and contextual approaches", en The nature of vocabulary acquisition, Margaret G. McKeown y Mary E. Curtis, (eds), Hillsdale, Lawrence Erlbaum Associates, 107-127.

San Mateo Valdehíta, Alicia (2013): "El conocimiento productivo y receptivo del vocabulario en L2: Análisis de la efectividad de tareas de reconocimiento y de producción", en Revista Nebrija de Lingüistica Aplicada a la Enseñanza de Lenguas, 14. [en línea]: http:// www.nebrija.com/revista-linguistica/el-conocimiento-productivo-y-receptivo-del-vocabulario-en-12 [consultado: 22 mayo 2018]

Sanjuán Álvarez, Marta (2008): "La adquisición del léxico como elemento central del currículo de español/L2 para inmigrantes", en Actas de las I Jornadas sobre Lenguas, Currículo y Alumnado Inmigrante, Ballano, Inmaculada (coord.), Bilbao, Universidad de Deusto, 173-186.

Sautermeister, Christine (1989): "Pour une meilleure compétence lexicale", en Lexiques, Ibrahim Andrew M. (coord.), Paris, Le Français dans le monde, 122-133.

Swaffer, Janet K. (1985): "Reading authentic texts in a foreign language: A cognitive model", en The Modern Language Journal, 69 (1), 15-34.

Swain, Michael (1995): "Three functions of output in second language learning", en Principles and Practice in Applied Linguistics, Guy Cook y Barbara Seidlhofer (eds.), Oxford, Oxford University Press.

Wallace, Michael (1982): Teaching Vocabulary, Oxford, Heinemann Educational Books. 